\title{
Luminescence Temperature Antiquenching of Water-Soluble CdTe Quantum Dots: Role of the Solvent
}

\author{
Sander F. Wuister, ${ }^{*}$ Celso de Mello Donegá, and Andries Meijerink \\ Contribution from the Debye Institute, Condensed Matter and Interfaces, Utrecht University, \\ Post Office Box 80 000, 3508 TA Utrecht, The Netherlands
}

Received March 29, 2004; E-mail: S.F.Wuister@phys.uu.nl

\begin{abstract}
Luminescence temperature antiquenching (LTAQ) is observed for water-soluble CdTe quantum dots (QDs) capped with aminoethanethiol (AET). The efficient exciton emission (quantum efficiency of $\sim 40 \%$ at $300 \mathrm{~K}$ ) is quenched almost completely as the QD solutions are cooled to below $230 \mathrm{~K}$ and is fully recovered around $270 \mathrm{~K}$ upon warming up to room temperature (LTAQ). Temperature-dependent lifetime measurements show that the quenching rate is high, resulting in an on/off behavior. No LTAQ is observed for CdTe QDs capped with aminoundecanethiol (AUT). The LTAQ is explained by the influence of solvent freezing on the surface of the QD core. Freezing of the solvation water molecules surrounding the QD will induce strain in the capping shell, due to the interaction between water and the charged heads of the capping molecules. Short carbon chains (AET) will propagate the strain to the QD surface, creating surface quenching states, whereas long and flexible chains (AUT) will dissipate the strain, thus avoiding surface distortion. Freezing-point depression by the addition of methanol results in a lowering of the transition temperature. Additional support is provided by the size dependence of the LTAQ: smaller particles, with higher local ionic strength due to a higher density of charged $\mathrm{NH}_{3}{ }^{+}$surface groups, experience a lower transition temperature due to stronger local freezing-point depression.
\end{abstract}

\section{Introduction}

Quantum dots (QDs) are an interesting class of materials since their electronic and optical properties mark a transition between bulk material and molecules. ${ }^{1,2}$ Especially the variation of the band gap with the size of the crystal has attracted much attention. Efficiently luminescing semiconductor QDs can be used in applications such as biological labels, ${ }^{3,4}$ quantum dot lasers, ${ }^{5}$ and LEDs. ${ }^{6}$ Monodisperse and highly luminescent II-VI QDs of CdSe and CdTe can currently be prepared via different hightemperature syntheses. ${ }^{7-10}$ The choice of the capping molecules used in the synthesis of the QDs is subtle but crucial for the formation of stable and highly luminescent semiconductor QD suspensions. ${ }^{11}$

The role of the capping molecules is 3 -fold. First, the capping molecules function as passivating agents for surface states. The

(1) Alivisatos, A. P. J. Chem. Phys. 1996, 100, 13226-13239.

(2) Murray, C. B.; Kagan, C. R.; Bawendi, M. G. Annu. Rev. Mater. Sci. 2000, 30, 545-610.

(3) Bruchez, M., Jr.; Moronne, M.; Gin, P.; Weiss, S.; Alivisatos, A. P. Science 1998, 281, 2013-2016.

(4) Dubertret, B.; Skourides, P.; Norris, D. J.; Noireaux, V.; Brivanlou, A. H.; Libchaber, A. Science 2002, 298, 1759-1762.

(5) Klimov, V. I.; Mikhailovsky, A. A.; Xu, S.; Malko, A.; Hollingsworth, J. A.; Leatherdale, C. A.; Eisler, H.; Bawendi, M. G. Science 2000, 290, 314317.

(6) Coe, S.; Woo, W.-K.; Bawendi, M. G.; Bulovic, V. Nature 2002, 420 , 800-803.

(7) Peng, Z. A.; Peng, X. J. Am. Chem. Soc. 2002, 124, 3343-3353.

(8) Talapin, D. V.; Rogach, A. L.; Shevchenko, E. V.; Kornowski, A.; Haase, M.; Weller, H. J. Am. Chem. Soc. 2002, 124, 5782-5790.

(9) de Mello Donegá, C.; Hickey, S. G.; Wuister, S. F.; Vanmaekelbergh, D.; Meijerink, A. J. Phys. Chem. B 2003, 107, 489-496.

(10) Talapin, D. V.; Haubold, S.; Rogach, A. L.; Kornowski, A.; Haase, M.; Weller, H. J. Phys. Chem. B 2001, 105, 2260-2263.

10.1021/ja048222a CCC: $\$ 27.50$ @ 2004 American Chemical Society surface-to-volume ratio of nanocrystals is large, and many luminescence quenching processes occur at the crystal surface. To prevent nonradiative recombination at surface sites, capping molecules cover the QD surface, passivating dangling bonds. Second, the growth kinetics of the QDs are determined by the affinity of the precursors for the capping molecules and the stability of the capping ligand precursor intermediate complex (e.g., TOP-Se). If the complex is too stable, no QD formation occurs. If the affinity with the capping molecules is too weak, the control over the crystal growth is poor, resulting in uncontrolled growth and formation of large particles (outside the quantum size regime). Third, the capping molecules prevent the QDs from aggregation via sterical hindrance (e.g., long aliphatic chains $)^{9-11}$ or by charge stabilization (e.g., $\mathrm{COO}^{-}$or $\mathrm{NH}_{3}{ }^{+}$groups at the surface). ${ }^{12}$

Although the role of the capping molecules is critical for the creation of a luminescent core, research on QDs has mainly focused on the properties of the semiconductor core. The capping layer of organic molecules is often regarded as an energy barrier to confine the exciton to the semiconductor core. The question if and how the capping molecules play an active role in the passivation and surface reconstruction of the QD core has recently been addressed for CdSe QDs capped with aliphatic amines. ${ }^{13}$ Evidence was presented for a strong influence

(11) Talapin, D. V.; Rogach, A. L.; Kornowski, A.; Haase, M.; Weller, H. Nano Lett. 2001, 1, 207-211.

(12) Wuister, S. F.; Swart, I.; Van Driel, F.; Hickey, S. G.; de Mello Donegá, C. Nano Lett. 2003, 3 503-507.

(13) Wuister, S. F.; Van Houselt, A.; de Mello Donegá, C.; Vanmaekelbergh, D.; Meijerink, A. Angew. Chem., Int. Ed. 2004, 43, 3029-3033.

J. AM. CHEM. SOC. 2004, 126, 10397-10402 - 10397 
of a phase transition in the capping molecule's shell on the luminescence behavior of highly luminescent CdSe QDs. A sharp increase in lifetime and quantum efficiency was observed when diluted samples of CdSe QDs were heated from 200 to $300 \mathrm{~K}$. This unusual luminescence behavior is called luminescence temperature antiquenching (LTAQ). ${ }^{13}$ The transition temperature for the recovery of the luminescence was found to increase with the chain length of the alkylamine capping molecule (from C6 to C18), consistent with a phase transition in the capping layer. ${ }^{13}$

In this paper LTAQ is reported for highly luminescent watersoluble CdTe QDs capped with aminoethanethiol (AET). Cooling of the CdTe QDs aqueous solutions below $230 \mathrm{~K}$ results in an almost complete quenching of the exciton emission, which fully recovers at $270 \mathrm{~K}$ upon heating to room temperature. The transition temperature is dependent on the size of the QD. For CdTe QDs capped with aminoundecanethiol (AUT) no LTAQ is observed. Experimental evidence is presented which shows that a phase transition in the solvent (water) around the QDs is responsible for the LTAQ of the CdTe QDs.

\section{Experimental Section}

Materials. Dimethylcadmium (99.9\%) was purchased from ARC Technologies. Tellurium $(99.999 \%,<250 \mu \mathrm{m})$ was purchased from Heraeus. Aminoundecanethiol (AUT, +90\%) was purchased from Dojindo. Dodecylamine (DDA; 98\%), trioctylphosphine (TOP; techn. 90\%), aminoethanethiol (AET, 98\%), methanol, and chloroform were purchased from Aldrich. DDA was dried for several hours under vacuum at $100{ }^{\circ} \mathrm{C}$ before use.

Synthesis of DDA-Capped CdTe QDs. DDA-capped QDs were prepared following the method reported by Wuister et al. ${ }^{14}$ In a threenecked flask $10 \mathrm{~g}$ of dry DDA and $7 \mathrm{~mL}$ of TOP were heated to 50 ${ }^{\circ} \mathrm{C}$. To this solution $0.22 \mathrm{~g}(1.54 \mathrm{mmol})$ of $\mathrm{Cd}(\mathrm{Me})_{2}$ in $7 \mathrm{~mL}$ of TOP and $0.16 \mathrm{~g}(1.25 \mathrm{mmol})$ of Te powder were added. $\mathrm{A} \mathrm{Cd} / \mathrm{Te}$ ratio of about 1.2 was used in all syntheses performed. The reaction mixture was heated to $145{ }^{\circ} \mathrm{C}$ for $3 \mathrm{~h}$ to yield green-emitting QDs $(d=2.7$ $\mathrm{nm})$. Orange-emitting $(d=3.0 \mathrm{~nm})$ QDs were prepared by heating the reaction mixture to $185^{\circ} \mathrm{C}$ for $2 \mathrm{~h}$. Red-emitting QDs $(d=3.4 \mathrm{~nm})$ were grown in two steps. First QDs were prepared by heating a TOP/ DDA mixture containing $0.22 \mathrm{~g}$ of $\mathrm{Cd}(\mathrm{Me})_{2}$ and $0.16 \mathrm{~g}$ of Te to 200 ${ }^{\circ} \mathrm{C}$ for $4 \mathrm{~h}$. The reaction mixture was then cooled to room temperature and $1.25 \mathrm{~mL}$ of a $0.3 \mathrm{M} \mathrm{TOP}-\mathrm{Te}$ solution (prepared by dissolving Te in TOP overnight at $200{ }^{\circ} \mathrm{C}$ ) was added. The resulting mixture was subsequently heated slowly to $185^{\circ} \mathrm{C}$ for $2 \mathrm{~h}$.

Synthesis of Aminoethanethiol-Capped CdTe QDs by Capping Exchange. To prepare water-soluble CdTe QDs with a positively charged capping, ${ }^{12} 50 \mu \mathrm{L}$ of the crude solution of CdTe QDs in the TOP/DDA coordinating mixture was dissolved in $5 \mathrm{~mL}$ of chloroform. Subsequently $50 \mu \mathrm{L}$ of $0.5 \mathrm{M}$ methanolic solution of AET was added. The stronger affinity of the thiol group (of AET) with the CdTe surface in comparison to the affinity of the amine group of DDA will cause a capping exchange of DDA by AET: the change of a surface with aliphatic chains (of DDA) to a surface with amine groups (of AET) causes flocculation of the QDs in chloroform. Subsequently $5 \mathrm{~mL}$ of deaerated ultrapure water $(16 \mathrm{M} \Omega \cdot \mathrm{cm})$ was added to the suspension, resulting in a two-phase system (water above chloroform). Upon shaking, the flocculated QDs were dissolved into the water phase, forming a clear suspension. The same procedure was followed for the preparation of AUT-capped QDs.

Apparatus. Emission spectra were recorded on a SPEX Fluorolog spectrofluorometer, equipped with two monochromators (double grating,

(14) Wuister, S. F.; Van Driel, F.; Meijerink, A. Phys. Chem. Chem. Phys. 2003, $5,1253-1258$.

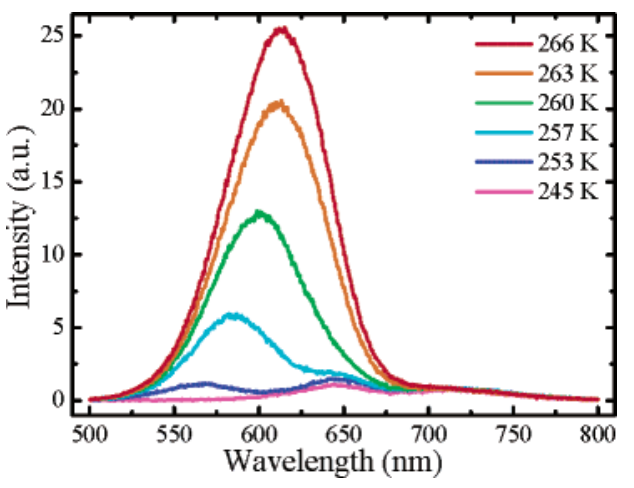

Figure 1. Temperature-dependent emission spectra $\left(\lambda_{\mathrm{ex}}=400 \mathrm{~nm}\right)$ of AETcapped CdTe QDs $(d=3.4 \mathrm{~nm})$ in water at temperatures between 245 and $266 \mathrm{~K}$.

$0.22 \mathrm{~m}$, SPEX 1680, model F2002) and a $450 \mathrm{~W}$ xenon lamp as the excitation source. Emission spectra were also recorded in this setup by collecting the emission with an optical fiber connected to a $0.25 \mathrm{~m}$ Acton Research monochromator fitted with a Princeton Instruments liquid nitrogen-cooled CCD camera. Luminescence lifetime measurements were performed with a Pico Quant picosecond laser $\left(\lambda_{\mathrm{ex}}=406\right.$ $\mathrm{nm}, 2.5 \mathrm{MHz})$ and monochromator (1350 lines $\mathrm{mm}^{-1}$ grating, blazed at $500 \mathrm{~nm}$ ) in combination with a fast Hamamatsu photomultiplier tube (H5738P-01) for light detection. Luminescence decay curves were obtained by pulse height analysis by use of a Time Harp 100 computer card. The ratio of stop to start pulses was kept low (below 0.05) to ensure good photon counting statistics. Temperature-dependent PL emission and lifetime measurements were recorded on a liquid helium flow cryostat. Samples were first cooled to $\sim 200 \mathrm{~K}$ and slowly warmed to room temperature (typical heating rates are $\sim 0.2 \mathrm{~K} / \mathrm{min}$.).

\section{Results and Discussion}

3.1. LTAQ of Water-Soluble CdTe QDs. Water-soluble, aminoethanethiol- (AET-) capped CdTe QDs show strong exciton emission at room temperature with quantum efficiencies up to $60 \% .^{12}$ In Figure 1 the temperature dependence of the emission spectra is depicted for red-emitting AET-capped CdTe QDs $(d=3.4 \mathrm{~nm})$ in water from 245 to $266 \mathrm{~K}$. At 266 $\mathrm{K}$ an emission band around $615 \mathrm{~nm}$ is observed that is ascribed to the exciton emission of the CdTe QDs. A weak emission band centered around $725 \mathrm{~nm}$ also observed in Figure 1. This weak emission band ( $\sim 3 \%$ of the total emission intensity) is ascribed to a defect-related emission of CdTe. ${ }^{14}$ The intensity of this defect-related emission is temperature-independent in the 245-266 K range. The intensity of the exciton peak, however, decreases rapidly with the temperature until it is almost totally quenched at $245 \mathrm{~K}$. The emission intensity does not recover when the sample is cooled further to $4 \mathrm{~K}$. As the sample is allowed to warm, a peak around $555 \mathrm{~nm}$ appears (from 250 $\mathrm{K}$ on) that strongly increases in intensity with temperature. This emission is ascribed to the recovered exciton emission of CdTe QDs. The emission intensity is completely recovered when the temperature reaches $266 \mathrm{~K}$, just below the melting point of pure water. This surprising LTAQ behavior of the exciton emission of CdTe QDs is reproducible and fully reversible. The behavior of the luminescence intensity for cooling and heating are identical (no hysteresis has been observed). The LTAQ can also be visually observed. Figure 2 illustrates the LTAQ with three photographs of a frozen aqueous solution of green-emitting CdTe QDs $(d=2.7 \mathrm{~nm})$ under $365 \mathrm{~nm}$ excitation (a real-time movie is also available as Supporting Information). In Figure 

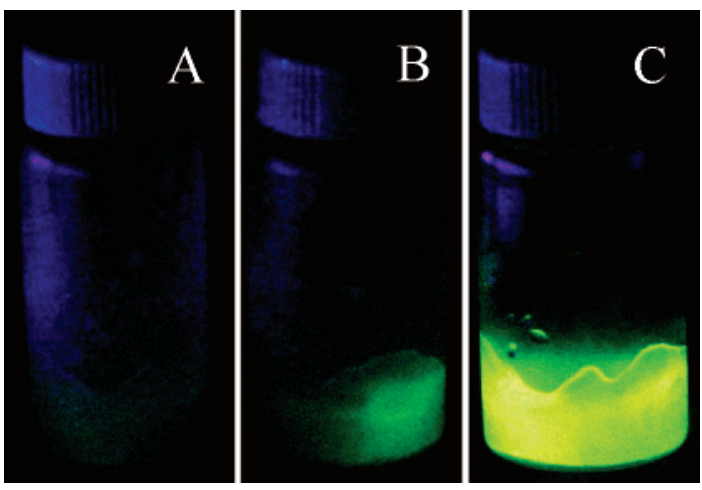

Figure 2. Photographs of a vial containing an aqueous solution of greenemitting CdTe QDs capped with AET $(d=2.7 \mathrm{~nm})$ in ice illuminated with a handheld mercury lamp $(365 \mathrm{~nm})$ at $\sim 77 \mathrm{~K}(\mathrm{~A})$ and just below $273 \mathrm{~K}$ (C). Panel B shows a vial warmed at the right-hand side (a real-time movie is also available as Supporting Information).

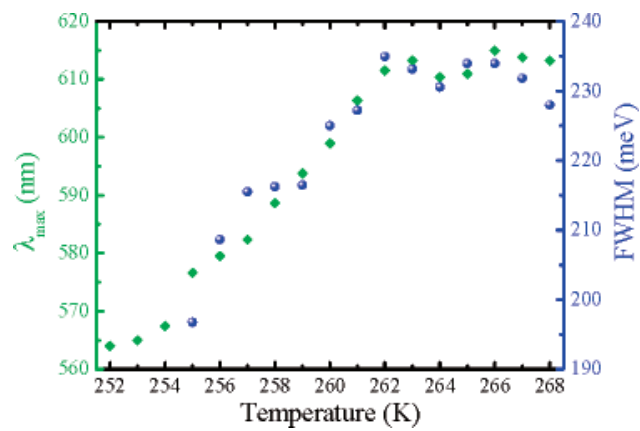

Figure 3. Emission maximum (green diamonds) and fwhm (blue circles) of the exciton emission of $3.4 \mathrm{~nm}$ AET-capped CdTe QDs in water as function of sample temperature.

$2 \mathrm{~A}$ the sample temperature is close to $77 \mathrm{~K}$ (thermal equilibrium with liquid $\mathrm{N}_{2}$ ), illustrating the complete quenching of the QD emission. Figure $2 \mathrm{C}$ shows the same sample just below the melting point of ice. The exciton emission of the green-emitting QDs has fully recovered. Figure 2B shows the intermediate case where only the right part of the vial was warmed, resulting in a half bright/half dark sample.

Figure 1 shows that as the temperature of the CdTe QDs sample is raised, not only the intensity of the exciton emission increases but also its maximum shifts toward lower energy (longer wavelengths). In Figure 3 (green diamonds) the maximum of the exciton emission peak of CdTe QDs is plotted versus the sample temperature. The exciton emission peak is observed to shift to lower energies by $175 \mathrm{meV}$ (from 564 to $614 \mathrm{~nm}$ ) from 252 to $263 \mathrm{~K}$. No further shift of the emission maximum occurs when the sample temperature is raised above $263 \mathrm{~K}$. The change of the emission color from green/yellow to orange/red is also noted by eye during the experiment. Furthermore, the full width at half-maximum (fwhm) of the exciton emission peak increases with temperature in the same range where the emission peak shifts to the red (from 255 to $263 \mathrm{~K}$; Figure 3, blue dots). Note that both effects occur over a very narrow temperature range (less than $10 \mathrm{~K}$ ).

Figures 1 and 3 indicate that the LTAQ of the AET-capped CdTe QDs is dependent on the crystal size. First the smallest QDs in the ensemble (largest band gap) start to emit. This results in a strongly blue-shifted emission band (compared to the emission at room temperature). In view of the Gaussianlike size distribution in a QD sample, only a small fraction of very small

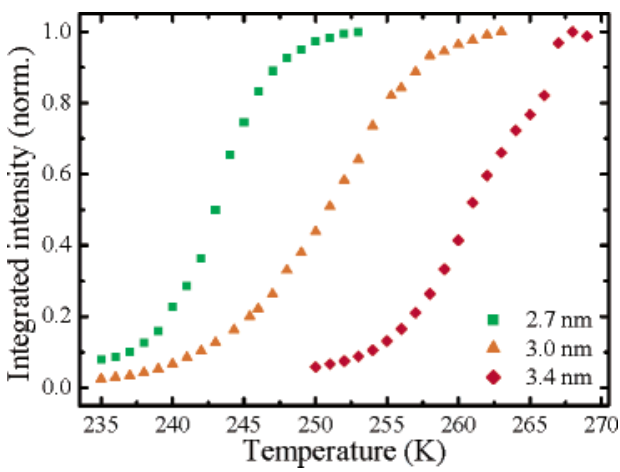

Figure 4. Normalized integrated intensity of the emission spectrum $\left(\lambda_{\mathrm{ex}}\right.$ $=400 \mathrm{~nm}$ ) for three different sizes $(2.7,3.0$, and $3.4 \mathrm{~nm})$ of AET-capped CdTe QDs in water as function of sample temperature.

particles emitting around $570 \mathrm{~nm}$ is present in the ensemble. This results in a low emission intensity at the blue side of the room-temperature emission spectrum. As the sample temperature increases further also larger QDs start to emit and contribute to the total emission, resulting in a red shift of the exciton emission and a higher luminescence intensity. The increase of the fwhm with temperature is consistent with a size-dependent quenching of the exciton luminescence. At temperatures higher than 263 $\mathrm{K}$ the fwhm and the position of the emission maximum remain constant, indicating that all the different sizes of QDs contribute to the total emission peak at $268 \mathrm{~K}$.

To confirm that the LTAQ of AET-capped CdTe QDs is indeed size-dependent, three different sizes of QDs were prepared, recapped with AET, and dissolved in water. The temperature dependence (from 235 to $270 \mathrm{~K}$ ) of the (normalized) integrated emission intensity of these CdTe QD samples is shown in Figure 4. A sharp transition (only $10 \mathrm{~K}$ from total quenching to total recovery) is observed for all three sizes. The red diamonds give the integrated intensity of the emission spectra depicted in Figure 1 (for particles with a diameter of $3.4 \mathrm{~nm}$ ). The total intensity increases sharply slightly above 260 $\mathrm{K}$. The S-shape of the curve reflects the size dispersion of the sample of QDs. Since only few relatively small and large particles are present, the contribution to the change in the total intensity is small in the low- and high-temperature regime of the curve. The particles with sizes close to the average size of the sample are abundantly present. As the temperature at which these particles start to emit is reached, a rapid change in the total intensity with temperature is observed. The steepness of the transition is dependent on the size distribution: as the size distribution decreases, the steepness of the transition increases. For smaller sizes $(2.7$ and $3.0 \mathrm{~nm})$ the same type of S-shaped curve is obtained. However, the transition temperature (the temperature at which the intensity has increased to $50 \%$ of the total intensity) decreases with decreasing size. This temperature behavior of the different CdTe samples confirms the size dependence of the LTAQ observed within an ensemble of CdTe QDs: for CdTe QDs with smaller sizes, the transition occurs at lower temperatures.

The luminescence decay curves can provide information about the decay kinetics of the CdTe exciton emission. ${ }^{14} \mathrm{CdTe}$ QDs capped with AET in water have a monoexponential decay with a (radiative) decay time close to $20 \mathrm{~ns}$ at room temperature. ${ }^{12}$ Figure 5 shows the luminescence decay curves of AET-capped 


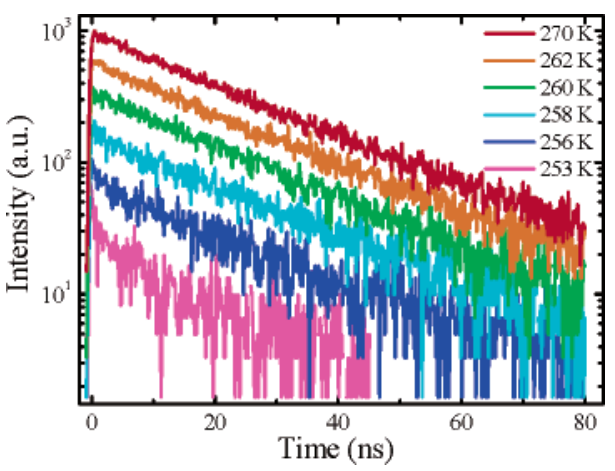

Figure 5. Temperature-dependent luminescence decay curves $\left(\lambda_{\mathrm{ex}}=400\right.$ $\mathrm{nm}$, measured at the emission maximum) of AET-capped CdTe QDs $(d=$ $3.4 \mathrm{~nm}$ ) in water.

CdTe QDs (3.4 nm in size) measured at the maximum of the exciton emission as a function of temperature. The area under the luminescence decay curve of the exciton emission increases with temperature in the same way as the integrated intensity determined from Figure 1. This is expected as the integral of the luminescence decay curves is proportional to the total light output and therefore to the photoluminescence quantum efficiency.

The decay curves at temperatures above $256 \mathrm{~K}$ are monoexponential and have similar decay times ( $\sim 20 \mathrm{~ns})$, whereas those measured at the lowest temperatures ( 253 and $256 \mathrm{~K}$ ) show a faster initial decay. The monoexponential character of the decay at higher temperatures indicates that purely radiative decay dominates the excitonic emission. The similar slopes also indicate that the nature of the dominant radiative process does not change during temperature increase. No shortening of the decay time is observed here as was detected for the LTAQ of alkylamine-capped CdSe QDs. For CdSe QDs a decrease of $30 \%$ of the room-temperature intensity is observed. ${ }^{13}$ Here the quenching is very effective, leading to a complete quenching of the exciton emission intensity, leaving only a weak defectrelated emission to be observed below the transition temperature. This indicates that a high nonradiative decay rate is associated with the quenching of the exciton emission. This results in an on/off behavior of the exciton luminescence. If the particle is on, the luminescence is dominated by radiative decay. ${ }^{12,15,16}$ Below the transition temperature (of the individual particle) the luminescence of a given QD is completely quenched (the off state). Since the particle is completely dark, it does not contribute to the luminescence and is therefore not detected in the luminescence decay curve. Consequently, luminescence lifetime determined from the luminescence decay curve does not change, since only the QDs in the on state (with a monoexponential decay) contribute to the luminescence signal.

3.2. Mechanism behind LTAQ. The LTAQ of the luminescence from CdTe QDs in water is more pronounced than the LTAQ observed from CdSe QDs reported previously. ${ }^{13}$ Before we discuss the mechanism behind the presently reported LTAQ, it is important to outline the differences between the two systems and to give a brief description of the mechanism proposed for LTAQ in CdSe QDs.

The system studied here (AET-capped CdTe QDs in water) differs in several aspects from the previously reported system

(15) Ebenstein, Y.; Mokari, T.; Banin, U. Appl. Phys. Lett. 2002, 80, 40334035 . where LTAQ was observed (alkylamine-capped CdSe QDs in toluene). In addition to a different semiconductor core material, also the capping and solvent molecules differ. Here, a thiol instead of an amine group is bound to the QD surface and a protonated amine group instead of an hydrophobic alkyl chain will interact with the solvent molecules. This makes the CdTe QD soluble in water instead of organic solvents. For CdSe QDs (in toluene) the solvent is still a liquid, while for AET-capped CdTe LTAQ is observed in the solid phase (ice).

The mechanism behind LTAQ for CdSe QDs is discussed in detail in ref 13. In brief, the model for LTAQ considers the influence of a phase transition in the capping layer on surface states of the QD. Total energy minimization calculations for TOPO-capped CdSe QDs have shown that the surface Cd and Se atoms shift slightly from their regular lattice position. ${ }^{17}$ This surface relaxation minimizes the energy, and it also removes surface states that are present in the band gap for the unrelaxed surface. Surface states in the band gap act as quenching centers, and to obtain high luminescence quantum yields, removal of these surface states by surface relaxation is essential. The LTAQ in CdSe QDs is explained by a phase transition in the layer of capping molecules at the QD surface. The long aliphatic chains of the alkylamine capping molecules have a strong interaction. In a low-temperature phase the stacking of the capping molecules induces strain to the relaxed QD surface and the surface atoms are removed from their positions. As a result, surface quenching states are created that lower the luminescence quantum yield. At higher temperatures the increased mobility of the capping molecules in a higher temperature phase lifts the strain on the surface and allows for surface relaxation, resulting in the recovery of the high luminescence quantum yield.

For AET-capped CdTe QDs a similar mechanism is considered to be responsible for the LTAQ of the exciton emission. CdTe QDs are capped with a short-chain thiol amine with a positively charged amine group. As a CdTe sample in water is cooled below $273 \mathrm{~K}$ the water freezes. The $\mathrm{NH}_{3}{ }^{+}$groups of the AET molecules that have strong interaction with the solvent will be incorporated in the solid. This strongly reduces their mobility, and strain is induced to the QD surface. As a result the relaxation of surface atoms of the CdTe QD is hampered and surface states in the band gap are created due to the unrelaxed surface. Fast nonradiative decay at the surface states becomes possible and quenches the exciton luminescence efficiently (the off state). When the sample is warmed, the ice melts in the direct proximity of the QD. This liberates the $\mathrm{NH}_{3}{ }^{+}$groups from their fixed positions, releases the strain induced to the surface, and allows for relaxation of the CdTe surface. The QD will then start to efficiently luminesce again (the on state).

Although the freezing point of (pure) water is $273 \mathrm{~K}$, the transition temperatures observed for LTAQ of the CdTe QDs are lower, as can be seen from Figure 4. The reason for the difference between the phase transition temperature of the (pure) solvent and the transition temperature is due to charge located at the QD surface as $\mathrm{NH}_{3}{ }^{+}$groups from the AET capping molecules. The freezing point of water can be lowered by

(16) Fisher, B. R.; Eisler, H. J.; Stott, N. E.; Bawendi, M. G. J. Phys. Chem. B 2004, 108, 143-148.

(17) Leung, K.; Whaley, K. B. J. Chem. Phys. 1999, 110, 11012-11022. 


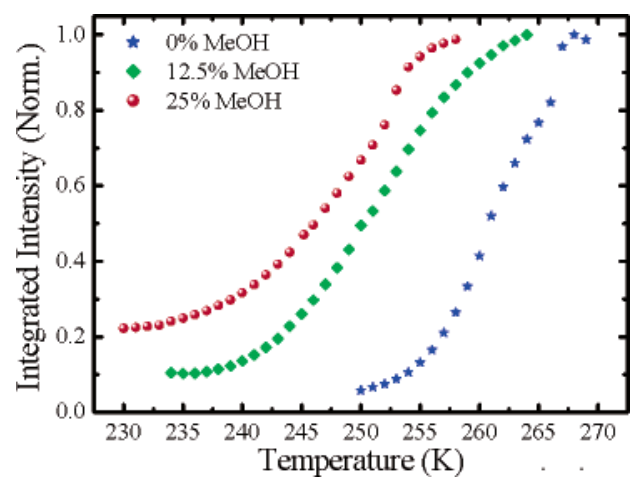

Figure 6. Integrated intensity of the emission spectra $\left(\lambda_{\mathrm{ex}}=400 \mathrm{~nm}\right)$ as a function of sample temperature of AET-capped CdTe QDs $(d=3.4 \mathrm{~nm})$ in water and in water with methanol (12.5 and 25 vol \%).

adding, for example, salts or alcohols (freezing-point depression). The AET-capped CdTe QDs are charge stabilized by protonated amine groups, leading to a high positive charge density at the QD surface. The presence of this charge at the QD surface will decrease the freezing-point temperature of the water in the direct proximity of the QD surface (local freezing-point depression). The freezing point of the solvent remains almost unaffected since the average ion concentration in the solvent is lower than at the positively charged QD surface due to the presence of the AET capping molecules. As pointed out above, it is the freezing of the solvent surrounding the QDs that hampers surface relaxation, bringing the QD in the off state. Due to the presence of charge at the QD surface, the local freezing point lowers the transition temperature to below $273 \mathrm{~K}$.

This local freezing-point depression can also give insight in the question why the LTAQ of AET-capped CdTe QDs is dependent on the QD size. For QDs the surface-to-volume ratio increases as the QD size decreases. Since AET is located at the QD surface, the charge density per volume is dependent on the size of the QD. Smaller QDs will therefore have a larger charge density per volume, which gives a stronger freezing-point depression in the local environment of the QD. Lower temperatures are needed to freeze the water in the direct surroundings of the AET at the QD surface. The overall effect is a lower quenching temperature of the exciton emission from the smaller QDs.

It is hard to obtain direct evidence for the presented model as it is not possible (in the present experiment) to probe the positions of the surface atoms of the CdTe QDs. To support the explanation presented above and provide evidence that indeed a liquid-solid phase transition in the solvent surrounding the QD is responsible for the LTAQ of CdTe QDs in water, additional experiments were performed.

To confirm that (local) freezing-point depression is responsible for the lowering of the transition temperature for LTAQ, the freezing point of the solvent can be lowered by addition of methanol. Figure 6 shows the temperature dependence of the integrated emission intensity of AET-capped CdTe QDs (the mean size is $3.4 \mathrm{~nm}$ ) without $\mathrm{MeOH}$ (blue stars), with $12.5 \%$ $\mathrm{MeOH}$ (green diamonds), and with $25 \% \mathrm{MeOH}$ (red circles). It was carefully verified that the addition of methanol to the solution of QDs did not result in flocculation. Figure 6 shows that the transition temperature decreases if more $\mathrm{MeOH}$ is added to the solution of QDs in water. Since the freezing point of the solution is lowered by the addition of $\mathrm{MeOH}$, the formation of

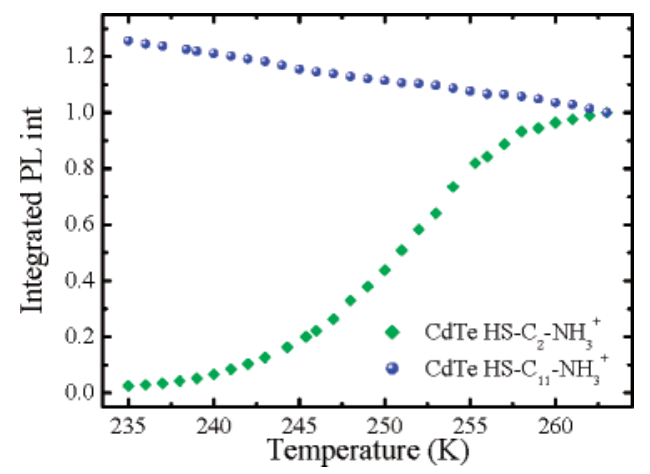

Figure 7. Temperature dependence of the integrated emission spectra $\left(\lambda_{\mathrm{ex}}\right.$ $=400 \mathrm{~nm})$ normalized to the intensity at $263 \mathrm{~K}$ of AET- $(\mathrm{C} 2$, green diamonds) and AUT- (C11, blue circles) capped CdTe QDs $(d=3.0 \mathrm{~nm})$ in water.

ice at the QD surface is now hampered not only by the charge of the AET molecules but also by the $\mathrm{MeOH}$ molecules in the solvent. This experiment provides additional proof that (a phase transition in) the solvent is responsible for the quenching of the exciton luminescence. The previous experiments show the important role of both the solvent and the capping. To obtain a deeper understanding of the role of the capping, the influence of the length of the carbon chain of the capping molecules was investigated. Two identical samples of orange-luminescing QDs (3.0 $\mathrm{nm}$ in diameter) were prepared, with AET $\left(\mathrm{C}_{2}\right)$ and aminoundecanethiol (AUT, $\mathrm{C}_{11}$ ) capping, respectively. Figure 7 shows the temperature dependence of the integrated emission intensity of AET-capped (green diamonds) and AUT-capped (blue circles) CdTe QDs in water.

For AET-capped QDs the above-reported LTAQ is observed. For AUT-capped QDs a gradual temperature quenching of the luminescence instead of a sharp antiquenching is observed. The integrated luminescence intensity of the AUT-capped CdTe QDs decreases with temperature in a similar manner as reported for TOPO-capped CdSe QDs ${ }^{18}$ and $\mathrm{CdSe}(\mathrm{ZnS})$-capped QDs. ${ }^{18,19}$ The absence of LTAQ for the AUT-capped QDs indicates that surface relaxation is not hampered by the freezing of the solvent surrounding the QD. The reason for the difference in temperature dependence is ascribed to the length of the aminoalkylthiol molecule. Since the chain length has increased considerably for the AUT molecules, fixation of the $\mathrm{NH}_{3}{ }^{+}$outer head does not hamper a relaxation of the surface $\mathrm{Cd}$ and $\mathrm{Te}$ atoms since the movement can be dissipated in the long $\left(\mathrm{C}_{11}\right)$ chain. This prevents the LTAQ and the CdTe cores from responding to the increasing temperature with a gradual decrease of the integrated intensity similar to that observed for $\mathrm{CdSe}(\mathrm{ZnS})$ QDs. The results shown in Figure 7 demonstrate the important role of both capping molecules and solvent on the luminescence behavior of semiconductor QDs.

\section{Conclusions}

The exciton luminescence of aminoethanethiol- $\left(\mathrm{C}_{2}-\right)$ capped CdTe QDs in water is completely quenched when they are cooled below $230 \mathrm{~K}$. The recovery of the luminescence is dependent on size and occurs over a very narrow temperature $(<10 \mathrm{~K})$ regime transition. The LTAQ is explained by the

(18) Crooker, S. A.; Barrick, T.; Hollingsworth, J. A.; Klimov, V. I. Appl. Phys. Lett. 2003, 82, 2793-2795.

(19) Walker, G. W.; Sundar, V. C.; Rudzinski, C. M.; Wun, A. W.; Bawendi, M. G.; Nocera, D. G. Appl. Phys. Lett. 2003, 83, 3555-3557. 
influence of a phase transition in the solvent on the surface relaxation of the CdTe QD. Local freezing-point depression by the charged amine groups at the QD surface shifts the transition temperature to lower values. Freezing-point lowering of the solvent by addition of methanol enhances this effect. No LTAQ is observed when a $\mathrm{C}_{11}$ amine thiol is used for capping. This demonstrates the crucial role of the solvent and capping molecules on the exciton luminescence properties of QDs.
Acknowledgment. This work was financially supported by Utrecht University within the Breedte-strategie program Physics of Colloidal Matter.

Supporting Information Available: One movie showing the LTAQ transition in real time for a sample of AET-capped CdTe QDs (MPEG). This information is available free of charge via the Internet at http://pubs.acs.org.

JA048222A 